\title{
Desempenho e divergência genética de cenoura 'Brasília' em função da procedência das sementes
}

\author{
Leilson C Grangeiro; Pascalle E de Azevêdo; Glauber Henrique de S Nunes; Mara Suyane M Dantas; \\ Claúdia A Cruz \\ UFERSA, Depto. Ciências Vegetais, C. Postal 137, 59625-900 Mossoró-RN; leilson@ufersa.edu.br; pascalleesco@yahoo.com.br; \\ glauber@ufersa.edu.br; marasuyane@gmail.com; mariahclaudia@hotmail.com
}

\section{RESUMO}

Os experimentos foram conduzidos na horta experimental da Universidade Federal Rural do Semi-Árido, Mossoró (RN), de agosto a novembro de 2007 e 2008 , com o objetivo de avaliar o desempenho produtivo e a divergência genética de cenoura 'Brasília' em função da procedência das sementes. O delineamento experimental utilizado foi em blocos casualizados completos, contendo oito tratamentos e quatro repetições. Os tratamentos foram constituídos pelas procedências das sementes de cenoura 'Brasília' das empresas Sakata, Embrapa Hortaliças, Tecnoseed, Hortec, Feltrin, Agristar/TopSeed, Seminis e Isla. Foram avaliados a massas secas da parte aérea e de raiz, produtividade total, comercial e não comercial de raízes, coloração das raízes (ângulo de cor $\mathrm{h}^{\circ}$, cromaticidade e luminosidade), sólidos solúveis e divergência genética. A cenoura 'Brasília' das empresas avaliadas apresentou desempenho produtivo e qualitativo dentro dos padrões da cultivar, para as condições de condução dos experimentos. No entanto, foram divergentes, em especial nas características de produção e coloração.

Palavras-chave: Daucus carota, produção, coloração de raiz.

\begin{abstract}
Performance and genetic divergence of 'Brasília' carrot depending on the source of seed

The experiments were carried out at the Universidade Federal Rural do Semi-Arido, in Mossoró, Rio Grande do Norte state, Brazil, from August to November 2007 and 2008. The performance and genetic divergence of carrot 'Brasília', depending on the source of the seeds was evaluated. The experimental design was of complete blocks design, with eight treatments and four replications. The treatments consisted of the sources of carrot seeds 'Brasilia', provided by Sakata, Embrapa Hortaliças, Tecnoseed, Hortec, Feltrin, Agristar/Topseed, Seminis and Isla. The variables dry mass of shoot and root, total, marketable and non- marketable roots yield, staining of the roots (hue, chroma and brightness), soluble solids and genetic divergence were measured. The 'Brasília' carrot presented the performance and quality standards within the cultivar, for the conditions of the experiments. However, it was different, especially in production and coloring characteristics.
\end{abstract}

Keywords: Daucus carota, production, root color.

(Recebido para publicação em 7 de junho de 2010; aceito em 7 de novembro de 2011) (Received on June 7, 2010; accepted on November 7, 2011)

\begin{abstract}
A cenoura (Daucus carota) é uma das mais importantes hortaliças de raiz comestível no Brasil, sendo produzidos anualmente cerca de 784 mil toneladas, em uma área de 26 mil hectares, com valor da produção de aproximadamente 340 milhões de dólares, equivalente a $4 \%$ do valor total da produção de hortaliças (EMBRAPA, 2011). Os maiores produtores são os municípios de Carandaí, São Gotardo e Maria da Fé (MG), Piedade, Ibiúna e Mogi das Cruzes (SP), Ponta Grossa e Marilândia (PR) e Irecê (BA).
\end{abstract}

No Brasil, até meados de 1980, devido à ocorrência da queima-das folhas (Alternaria dauci + Cercospora carotae + Xanthomonas campestris pv. carotae.), o cultivo de cenoura na época chuvosa de verão exigia a aplicação quase diária de fungicidas, chegando a 50 aplicações durante o seu ciclo, estimado em 120 dias. Consequentemente, baixas produtividades e elevados custos de produção gerados por aplicações excessivas de agrotóxicos tornavam baixa a rentabilidade da cultura. Assim sendo, devido aos fatores supracitados, o cultivo da cenoura em época chuvosa era limitado em área e produtividade, reduzindo a oferta do produto e inflacionando o seu preço. Os agrotóxicos e as sementes importadas eram os componentes que mais oneravam os custos finais da produção, e assim os lucros auferidos não estimulavam a expansão das áreas existentes e os baixos volumes produzidos não atendiam a demanda interna do produto (Vilela \& Borges, 2008).

O lançamento da cultivar de cenoura Brasília, pela Embrapa Hortaliças e ESALQ em 1981, mudou o agronegó- cio de cenoura no país, impulsionou a produção interna e regularizou o abastecimento ao decorrer do ano, pois tornou possível o cultivo em regiões antes não cultivadas, como por exemplo no Nordeste brasileiro e no verão nas demais regiões. É a principal cultivar de cenoura, representando aproximadamente $40 \%$ da área plantada ${ }^{1 *}$. Para atender essa produção, existe uma necessidade anual de sementes de aproximadamente 140 toneladas, que até então, era suprida inteiramente pela importação. A produção de sementes de cenoura de cultivares desenvolvidas no Brasil está concentrada na região sul do país, face à ocorrência de condições favoráveis no que se refere à indução ao florescimento por baixas temperaturas e/ ou fotoperíodo crescente.

Atualmente, grande parte das sele-

\footnotetext{
${ }^{1 *}$ Informações fornecidas por Dr. Jairo V. Vieira, Pesquisador da Embrapa Hortaliças.
} 
ções da cultivar Brasília que estão disponíveis no mercado está geneticamente descaracterizada, mostrando padrão varietal diferente do originalmente descrito em 1981. Esse fato é explicado pelas seleções realizadas pelas empresas produtoras de sementes, com o intuito de melhorar a qualidade das raízes pela redução da freqüência de características como por exemplo ombro verde e coloração interna pouco alaranjada, presentes na cultivar Brasília original. Os trabalhos de melhoramento conduzidos pelas empresas sementeiras resultaram em alta variabilidade presente nos lotes comercializados pelas respectivas instituições, possibilitando diferenciá-los da cultivar Brasília original. Sendo assim, pode-se afirmar que, para atender as mudanças e exigências do mercado consumidor, o desenvolvimento de programas de seleção visando melhorar a uniformidade dos atributos de qualidade da cv. Brasília ainda se constitui uma demanda prioritária de pesquisa (Andrade et al., 2003).

O presente trabalho teve como objetivo, avaliar o desempenho e a divergência genética de cenoura 'Brasília' em função da procedência das sementes.

\section{MATERIAL E MÉTODOS}

Os experimentos foram desenvolvidos na horta experimental da Universidade Federal Rural do Semiárido, Mossoró (RN) de agosto a novembro de 2007 e 2008 . O delineamento experimental utilizado foi em blocos casualizados completos com oito tratamentos e quatro repetições. Os tratamentos foram constituídos pelas procedências das sementes de cenoura 'Brasília' das empresas Sakata, Embrapa Hortaliças, Tecnoseed, Hortec, Feltrin, Agristar/ TopSeed, Seminis e Isla. Cada unidade experimental teve as dimensões de 1,2 $\mathrm{x} 1,8 \mathrm{~m}$, contendo nove fileiras longitudinais, no espaçamento de $20 \times 10 \mathrm{~cm}$, totalizando 108 plantas.

A adubação foi realizada de acordo com a análise de solo e recomendação de Cavalcanti (1998), utilizando-se em fundação $20 \mathrm{t} \mathrm{ha}^{-1}$ de esterco bovino, $30 \mathrm{~kg} \mathrm{ha}^{-1}$ de $\mathrm{N}, 60 \mathrm{~kg} \mathrm{ha}^{-1}$ de $\mathrm{P}_{2} \mathrm{O}_{5}$ e $40 \mathrm{~kg} \mathrm{ha}^{-1} \mathrm{de}_{2} \mathrm{O}$, nas formas de uréia, superfosfato simples e cloreto de potássio respectivamente. Em cobertura utilizaram-se $40 \mathrm{~kg} \mathrm{ha}^{-1}$ de $\mathrm{N}$ e $30 \mathrm{~kg} \mathrm{ha}^{-1}$ de $\mathrm{K}_{2} \mathrm{O}$, nas formas de uréia e cloreto de potássio, parcelados em duas aplicações aos 25 e 45 dias após a semeadura. Semanalmente, após o desbaste até duas semanas antes da colheita foi aplicado via foliar o fertilizante organomineral contendo: $10 \%$ de $\mathrm{N} ; 1 \%$ de $\mathrm{P}_{2} \mathrm{O}_{5} ; 1 \%$ de $\mathrm{K}_{2} \mathrm{O} ; 1 \%$ de $\mathrm{Ca} ; 0,5 \%$ de $\mathrm{Mg} ; 3 \%$ de S; $0,5 \%$ de B; $0,5 \%$ de $\mathrm{Mn} ; 0,005 \%$ de Mo; $1,5 \%$ de Zn e $6 \%$ de carbono orgânico, na dose de $2,5 \mathrm{~mL} \mathrm{~L}^{-1}$ de água. A colheita foi realizada aos 85 e 78 dias após semeadura, respectivamente no primeiro e segundo experimento. Para avaliação dos resultados, foi coletada uma amostra de 30 plantas competitivas da área útil da parcela.

As características avaliadas foram: massa seca da parte aérea (MSPA) e de raízes (MSR), produtividades total (PDT), comercial (PDC) e não comercial (PDNC), sólidos solúveis (SS), $\mathrm{pH}$ e coloração interna das raízes. A coloração foi estimada utilizando-se de um colorímetro Minolta Croma Meter CR 200b, calibrado em superfície de porcelana branca sob condições de iluminação e expressa no módulo L, a* e $\mathrm{b}^{*}$. Em seguida foram determinados os valores de cromaticidade (C), luminosidade (L) e ângulo de cor $\left(\mathrm{h}^{\circ}\right)$, segundo metodologia de Mcguire (1992). Também foi realizado estudo de divergência genética. Os dados foram submetidos a análises de variâncias isoladas para cada experimento, procedendo-se em seguida a análise conjunta dos experimentos. As médias de procedências foram comparadas pelo teste de Scott-Knott com 5\% de probabilidade (Scott \& Knott, 1974), e as médias de época foram comparadas pelo Teste-t de Student em nível de 5\% de probabilidade (Steel \& Torrie, 1981).

Para a divergência genética foram realizadas análises de variância univariada e posteriormente, análise de variância multivariada e aplicação do critério de Wilks a 5\% de probabilidade. A partir da matriz com as médias de cada característica para cada população e da matriz de variância-covariância residual, foram calculadas as distâncias generalizadas de Mahalanobis $\left(\mathrm{D}^{2}{ }_{\mathrm{ij}}\right)$. Realizou-se o diagnóstico de multi- colinearidade da matriz de correlação residual pelo método de Montgomery \& Peck (Cruz et al., 2004). A análise de agrupamento foi realizada com a matriz de distâncias de Mahalanobis entre as populações, utilizando o método UPGMA. Foi utilizado o critério de Singh para identificar a contribuição relativa de cada característica para a divergência genética.

\section{RESULTADOS E DISCUSSÃO}

Houve efeito significativo da interação tratamento (procedência das sementes) x épocas para as características massa seca de raiz (MSR), produtividade comercial (PDC) e produtividade não comercial (PNDC). Os fatores isolados época e tratamentos foram significativos para sólidos solúveis (SS), luminosidade (L), cromaticidade (C) e ângulo de cor $\left(h^{\circ}\right)$. Para a massa seca da parte aérea (MSPA) e produtividade total (PDT) apenas a época foi significativa.

A interação entre as populações de cenoura 'Brasília' (procedência das sementes) e épocas para as características MSR, PDC e PDNC, indica comportamento diferente das mesmas nas duas épocas avaliadas. A interação prevalecente para PDC e PDNC foi do tipo complexa, enquanto que MSR foi do tipo simples.

Uma vez detectada a interação, realizou-se o desdobramento de populações dentro das épocas de cultivo de avaliação. No experimento de 2007 , não houve diferença significativa entre os tratamentos para a produtividade comercial (PDC) e não comercial (PDNC). Em 2008, as sementes das empresas Agristar/Topseed e Isla proporcionaram PDC inferiores às demais. Entre as épocas, verificou-se que, com exceção da Isla e Seminis, as maiores produtividades foram obtidas em 2008 (Tabela 1).

Já para a PDNC, em 2008 a cenoura 'Brasília' da empresa Isla apresentou maior valor que as demais. Entre as épocas de cultivo, com exceção da semente da Tecnoseed e Isla, a maior PDNC foi verificada em 2007 para as sementes das demais empresas. A Tecnoseed não mostrou diferença significativa entre as duas épocas de cultivo, 
Tabela 1. Produtividade comercial (PDC), produtividade não comercial (PDNC) e massa seca de raiz (MSR) de cenoura, cv. Brasília, em função da procedência da semente e época de condução do experimento (non- marketable (PDNC) and marketable yield (PDC) and root dry mass (MSR) of carrot cv. Brasília, depending on seed source and moment of execution of the experiment). Mossoró, UFERSA, 2007 e 2008.

\begin{tabular}{|c|c|c|c|c|c|c|}
\hline \multirow{2}{*}{ Procedência da semente } & \multicolumn{2}{|c|}{ PDC $\left(\mathrm{kg} / \mathrm{m}^{2}\right)$} & \multicolumn{2}{|c|}{$\operatorname{PDNC}\left(\mathrm{kg} / \mathrm{m}^{2}\right)$} & \multicolumn{2}{|c|}{ MSR (g/planta) } \\
\hline & 2007 & 2008 & 2007 & 2008 & 2007 & 2008 \\
\hline Embrapa Hortaliças & $3,37 \mathrm{Ab}^{1}$ & $4,16 \mathrm{Aa}$ & $0,70 \mathrm{Aa}$ & $0,45 \mathrm{Bb}$ & $6,61 \mathrm{Ab}$ & $10,91 \mathrm{Aa}$ \\
\hline Hortec & $3,30 \mathrm{Ab}$ & $4,27 \mathrm{Aa}$ & $0,68 \mathrm{Aa}$ & $0,19 \mathrm{Bb}$ & $5,06 \mathrm{Bb}$ & $8,69 \mathrm{Ba}$ \\
\hline Tecnoseed & 3,31 Ab & $4,07 \mathrm{Aa}$ & $0,50 \mathrm{Aa}$ & $0,49 \mathrm{Ba}$ & $5,52 \mathrm{Bb}$ & $9,66 \mathrm{Ba}$ \\
\hline Sakata & 3,31 Ab & 3,95 Aa & $0,49 \mathrm{Aa}$ & $0,30 \mathrm{Bb}$ & $5,39 \mathrm{Bb}$ & $9,51 \mathrm{Ba}$ \\
\hline Feltrin & $2,83 \mathrm{Ab}$ & 4,17 Aa & $0,40 \mathrm{Aa}$ & $0,39 \mathrm{Bb}$ & $6,12 \mathrm{Ab}$ & $10,85 \mathrm{Aa}$ \\
\hline Agristar/Topseed & $3,22 \mathrm{Ab}$ & 3,63 Ba & $0,29 \mathrm{Aa}$ & $0,19 \mathrm{Bb}$ & $5,89 \mathrm{Bb}$ & $10,23 \mathrm{Ba}$ \\
\hline Seminis & 3,32 Aa & $3,84 \mathrm{Aa}$ & $0,20 \mathrm{Aa}$ & $0,24 \mathrm{Bb}$ & $5,81 \mathrm{Bb}$ & $9,84 \mathrm{Ba}$ \\
\hline Isla & $3,56 \mathrm{Aa}$ & $3,13 \mathrm{Bb}$ & $0,32 \mathrm{Ab}$ & $0,88 \mathrm{Aa}$ & $6,70 \mathrm{Ab}$ & $12,35 \mathrm{Aa}$ \\
\hline Média & 3,28 & 3,90 & 0,45 & 0,39 & 5,89 & 10,25 \\
\hline CV $(\%)$ & \multicolumn{2}{|c|}{11,67} & \multicolumn{2}{|c|}{61,33} & \multicolumn{2}{|c|}{12,94} \\
\hline
\end{tabular}

${ }^{1}$ Médias seguidas de mesma letra maiúscula na coluna e de mesma letra minúscula na linha, não diferem entre si pelo teste de Scott Knott $\mathrm{p}<0,05$ (means followed by same capital letter in the colums and small letter in the row do not differ through the Skott Knott test $\mathrm{p}<0.05$ ).

Tabela 2. Massa seca da parte aérea (MSPA), produtividade total (PDT), sólidos solúveis (SS), pH, cromaticidade (C), ângulo de cor ( $\mathrm{h}^{\circ}$ ) e luminosidade (L) de raízes de cenoura cv. Brasília, em função da época de condução do experimento (shoot dry mass (MSPA), total yield (PDT), solid solubles (SS), $\mathrm{pH}$, chromaticity $(\mathrm{C})$, hue $\left(\mathrm{h}^{\circ}\right)$ and luminosity $(\mathrm{L})$ of carrot cv. Brasília, depending on the moment of execution of the experiment). Mossoró, UFERSA, 2007 e 2008.

\begin{tabular}{cccccccc}
\hline Época & MSPA (g/planta) & PDT (kg/m $)$ & SS (\%) & pH & C & $\mathbf{h}^{\mathbf{0}}$ & L \\
\hline 2007 & $10,64 \mathrm{~B}^{1}$ & $3,72 \mathrm{~B}$ & $8,50 \mathrm{~A}$ & $6,47 \mathrm{~A}$ & $36,14 \mathrm{~B}$ & $62,22 \mathrm{~B}$ & $56,65 \mathrm{~A}$ \\
2008 & $13,27 \mathrm{~A}$ & $4,29 \mathrm{~A}$ & $7,92 \mathrm{~B}$ & $6,00 \mathrm{~B}$ & $42,77 \mathrm{~A}$ & $75,08 \mathrm{~A}$ & $55,30 \mathrm{~A}$ \\
\hline CV $(\%)$ & 25,82 & 11,16 & 7,71 & 6,14 & 10,98 & 5,83 & 4,81 \\
\hline
\end{tabular}

${ }^{1}$ Médias seguidas de mesma letra maiúscula na coluna não diferem entre si pelo Teste-t de Student $\mathrm{p}<0,05$ (means followed by same capital letter in the colums do not differ through the Test-t $\mathrm{p}<0.05$ ).

Tabela 3. Sólidos solúveis (SS), luminosidade (L), cromaticidade (C) e ângulo de cor $\left(\mathrm{h}^{\circ}\right)$, de cenoura cv. Brasília, em função da procedência da semente (soluble solids (SS), luminosity (L), chromaticity (C) and hue ( $\mathrm{h}^{\circ}$ ), of carrot cv. Brasília, depending on seed source). Mossoró, UFERSA, 2007 e 2008.

\begin{tabular}{lcccc}
\hline Procedência da semente & SS (\%) & $\mathbf{C}$ & $\mathbf{h}^{\mathbf{0}}$ & $\mathbf{L}$ \\
\hline Embrapa Hortaliças & $8,32 \mathrm{~A}^{1}$ & $38,64 \mathrm{~B}$ & $69,00 \mathrm{~A}$ & $54,56 \mathrm{~B}$ \\
Hortec & $8,01 \mathrm{~A}$ & $36,87 \mathrm{~B}$ & $71,31 \mathrm{~A}$ & $55,13 \mathrm{~B}$ \\
Tecnoseed & $8,10 \mathrm{~A}$ & $36,00 \mathrm{~B}$ & $72,00 \mathrm{~A}$ & $56,06 \mathrm{~B}$ \\
Sakata & $8,22 \mathrm{~A}$ & $39,21 \mathrm{~B}$ & $69,24 \mathrm{~A}$ & $55,00 \mathrm{~B}$ \\
Feltrin & $7,62 \mathrm{~B}$ & $36,53 \mathrm{~B}$ & $70,21 \mathrm{~A}$ & $54,56 \mathrm{~B}$ \\
Agristar/Topseed & $8,06 \mathrm{~A}$ & $42,00 \mathrm{~A}$ & $67,30 \mathrm{~B}$ & $57,23 \mathrm{~A}$ \\
Seminis & $8,90 \mathrm{~A}$ & $45,06 \mathrm{~A}$ & $66,20 \mathrm{~B}$ & $58,37 \mathrm{~A}$ \\
Isla & $8,41 \mathrm{~A}$ & $41,39 \mathrm{~A}$ & $67,00 \mathrm{~B}$ & $56,90 \mathrm{~A}$ \\
\hline CV (\%) & 7,71 & 10,98 & 5,83 & 4,81 \\
\hline
\end{tabular}

${ }^{1}$ Médias seguidas de mesma letra maiúscula na coluna não diferem entre si pelo teste de Scott-Knott, $\mathrm{p}<0,05$ (means followed by same capital letter in the colums do not differ through the Skott Knott test $\mathrm{p}<0.05$ ).

e a Isla a PDNC em 2008 foi superior. No experimento de 2007, embora não tenha ocorrido diferença significativa entre os tratamentos, a PDC variou de $2,83 \mathrm{~kg} \mathrm{~m}^{-2}\left(19,8 \mathrm{t} \mathrm{ha}^{-1}\right)$ para a cenoura 'Brasília' da empresa Feltrin a 3,56 kg $\mathrm{m}^{-2}\left(24,9\right.$ t ha $\left.^{-1}\right)$ da empresa Isla. A massa seca de raiz (MSR) no cultivo de 2008 foi superior a 2007 , independentemente da procedência da semente. Nos dois experimentos, a cenoura 'Brasília' das empresas Embrapa Hortaliças, Feltrin e
Isla apresentaram as maiores MSR, e as demais não diferiram estatisticamente (Tabela 1).

Com relação à produtividade total (PDT), no cultivo de 2008 foi obtida uma média de 4,29 $\mathrm{kg} \mathrm{m}^{-2}\left(30,0 \mathrm{t} \mathrm{ha}^{-1}\right)$, 


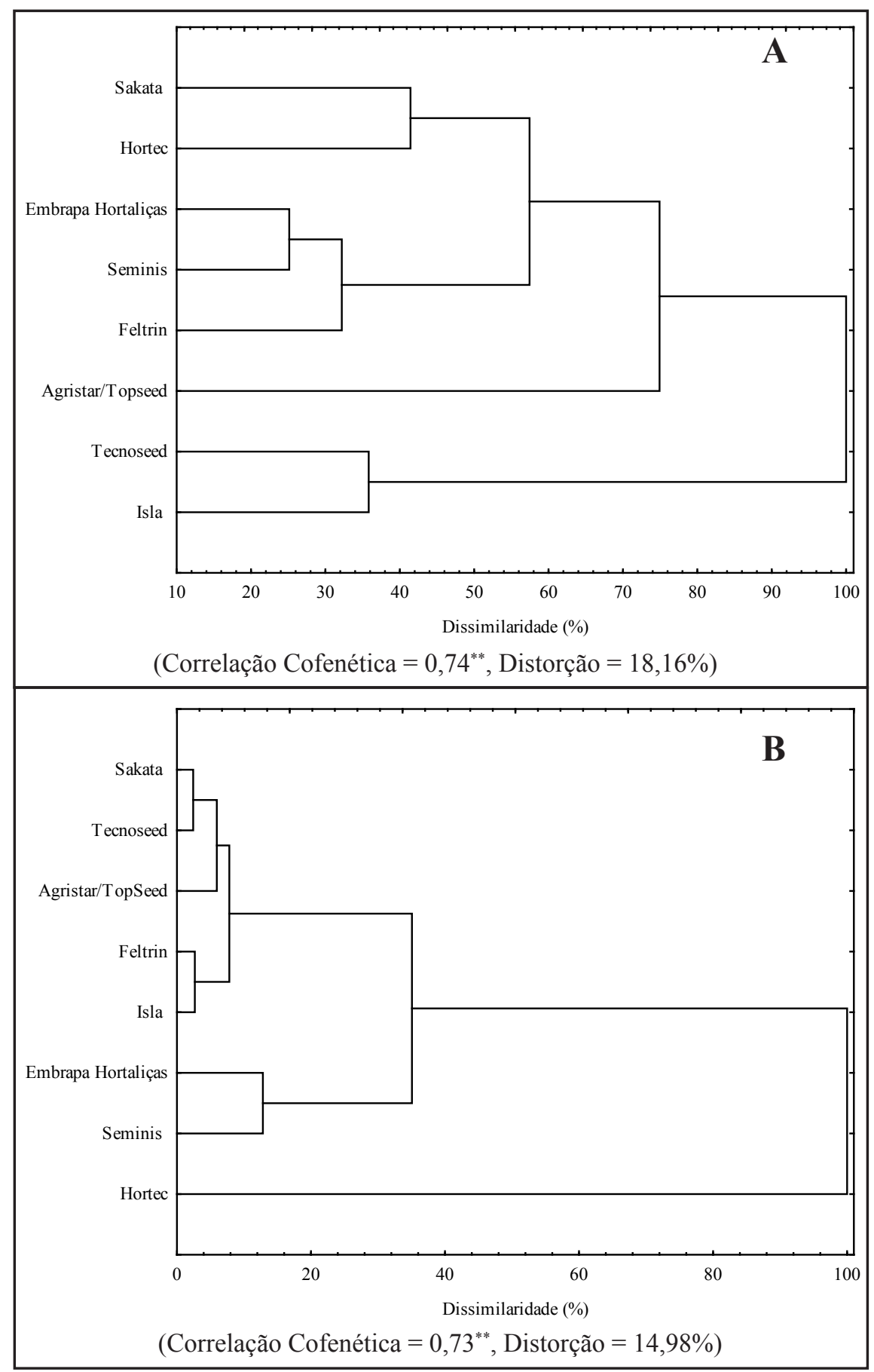

Figura 1. Dendrograma entre oito populações de cenoura 'Brasília' oriundas de diferentes empresas construídas pelo UPGMA a partir das distancias generalizadas de Mahalanobis (dendrogram among eight populations of carrot 'Brasilia' coming from different companies constructed by UPGMA from the Mahalanobis). Mossoró, UFERSA, 2007 e 2008.

superior a $3,72 \mathrm{~kg} \mathrm{~m}^{-2}\left(26,0 \mathrm{t} \mathrm{ha}^{-1}\right)$ do cultivo de 2007 (Tabela 2). O desempenho da cenoura 'Brasília', independentemente da procedência das sementes, pode ser considerado satisfatório nas condições em que foram conduzidos os experimentos, tendo em vista que em trabalhos anteriores conduzidos ambientes e espaçamentos no município de São José do Rio Pardo (SP), dos quais 'Brasília' das empresas Isla, SVS e Sakata, verificaram que em média não houve diferença significativa na produtividade comercial entre os materiais de cenoura 'Brasília' dentro de cada ambiente avaliado. No entanto, quando a avaliação foi feita entre os ambientes, observou-se variação na produtividade. Em outra situação, Clemente et al. (2006) avaliaram diferentes populações e cultivares de cenoura em cultivo orgânico, das quais cenoura 'Brasília' das empresas Horticeres, Hortec, Asgrow, Bionatur e da Embrapa Hortaliças, verificaram que todas as populações e cultivares tiveram bom desempenho comercial. No entanto, entre os materiais de cenoura 'Brasília', as das empresas Asgrow (39,4 t ha-1) e Embrapa Hortaliças $\left(42,5 \mathrm{t} \mathrm{ha}^{-1}\right)$ apresentaram melhor desempenho produtivo, sendo que as demais não diferiram estatisticamente.

Para a massa seca da parte aérea, houve apenas diferença entre as épocas de cultivo, com maior MSPA obtida no experimento de 2008 (Tabela 2). Um dos aspectos relevantes para este fato pode estar relacionado com o grau de maturação uniforme entre as plantas, uma vez que todos os materiais foram plantados e colhidos ao mesmo tempo.

Para os sólidos solúveis (SS), a cenoura 'Brasília' da empresa Feltrin apresentou valor inferior às demais, sendo que estas não diferiram entre si. Os resultados médios obtidos variaram de 7,62 a 8,90\% (Tabela 3). Com relação à época de cultivo, o experimento de 2007, proporcionou em média teor SS de $8,50 \%$, superior a $7,92 \%$ de 2008 (Tabela 2). Os teores médios de SS obtidos no presente trabalho estão na faixa dos encontrados em cultivares do tipo Brasília que podem variar de 4,5 a $12,5 \%$ (Lana \& Vieira, 2000). Pereira et al. (2008) também obtiveram valores de SS próximos aos aqui verificados, com variação entre 6,7 e 7,4\%, na ocasião da colheita.

Com relação à coloração interna da raiz, observou-se a formação de dois grupos, para o ângulo de cor $\left(\mathrm{h}^{\circ}\right)$, cromaticidade (C) e luminosidade (L). Para o ângulo de cor, o grupo formado pela cenoura 'Brasília' das empresas 
Tecnoseed, Hortec, Feltrin, Sakata e Embrapa Hortaliças foi superior ao das demais empresas. Portanto, a coloração interna (xilema) de raiz no primeiro grupo foi mais amarela em relação à do segundo grupo. Já os valores de cromaticidade e de luminosidade foram menores no primeiro grupo, reafirmando que apresentou uma coloração menos intensa, em relação ao que obteve valores de ângulo de cor menores (Tabela 3). Embora, a diferença entre os grupos de materiais de cenoura 'Brasília' com relação aos valores de ângulo de cor $\left(\mathrm{h}^{\circ}\right)$ tenha sido pequena, as cenouras das empresas Agristar/Topseed, Seminis e Isla apresentaram uma coloração interna (xilema) tendendo para o amarelo laranja.

Para a época de cultivo, o experimento de 2008 proporcionou valores de ângulo de cor $\left(\mathrm{h}^{\circ}\right)$ e cromaticidade (C) superiores a 2007 (Tabela 2). Não houve diferença significativa para a luminosidade (L), entre as épocas. A coloração interna e externa da raiz é uma característica importante, e que deve ser utilizada nos programas de melhoramento de cenoura, visando a criação de novas cultivares (Vieira \& Silva, 2008). Na produção de sementes, como a maioria das empresas utiliza o método semente-semente, não é feita a seleção das raízes com base na coloração interna (xilema), o que tem proporcionado uma desuniformidade no padrão de qualidade de cenoura 'Brasília' no mercado.

No estudo de divergência genética, em experimento conduzido em 2007, a análise de agrupamento a partir das distancias generalizadas de Mahalanobis proporcionou a formação de quatro grupos (Figura 1A). O primeiro formado pelas empresas Isla e Tecnossed, o segundo pela empresa Agristar/Topseed, o terceiro, pelas empresas Feltrin, Seminis e Embrapa Hortaliças. Por fim, o quarto grupo pelas empresas Sakata e Hortec.

No experimento de 2008, a análise de agrupamento propiciou três grupos de empresas. O primeiro foi constituído apenas pela Hortec. O segundo foi composto pelas empresas Embrapa Hortaliças e Seminis, enquanto que o terceiro foi composto pelas demais empresas (Figura 1B). Constatou-se, portanto, que o agrupamento foi extremamente diferente nos dois anos de avaliação.

Esse fato revela que o uso de caracteres morfológicos em estudos de divergência genética pode levar a resultados discrepantes. Um das razões é a presença da interação genótipo $\mathrm{x}$ ambiente, que dificulta a seleção e/ou recomendação, pois o desempenho de um determinado material é em função do genótipo e do ambiente (Vieira et al., 2005).

Com relação às contribuições relativas de cada característica para divergência genética verificou-se que no experimento de 2007, as características que mais contribuíram foram cromaticidade, ângulo de cor $\mathrm{h}^{\circ}$, luminosidade e produção comercial e não comercial. No experimento de 2008, quase toda divergência esteve concentrada nas características de produção com mais de 95\%, com destaque para as produções não comercial $(47,54 \%)$ e comercial $(35,51 \%)$.

Pelos resultados do estudo de divergência genética, pode-se constatar que as diferentes populações disponíveis para comercialização são divergentes, em especial nas características de produção e coloração interna das raízes. Considerando que a cultivar 'Brasília' é oriunda de uma população de ampla variabilidade genética e que o método de seleção utilizado na obtenção dessa cultivar (seleção de famílias de meio-irmãos) permite a conservação da variabilidade genética dentro da mesma (Silva et al., 2008), e que as empresas devem realizar seleção durante o processo de obtenção das sementes e produção de raízes, era esperada variabilidade entre as populações, indicando que as sementes comercializadas no mercado, obviamente, não correspondem geneticamente à população original. Todavia, deve ser enfatizado que, em todas as populações estudadas, as propriedades que tornaram a cultivar 'Brasília' um sucesso no agronegócio da cenoura no Brasil foram observadas.

A cenoura 'Brasília' das diferentes empresas apresentou desempenho satisfatório para as condições em que foram conduzidos os experimentos. No entanto, as mesmas foram divergentes, principalmente, com relação à produtividade e coloração interna das raízes.

\section{AGRADECIMENTOS}

À CAPES pela concessão da bolsa de mestrado para o segundo autor, e ao Dr. Jairo V. Vieira, da Embrapa Hortaliças, pelas informações e fornecimento de sementes de cenoura 'Brasília'.

\section{REFERÊNCIAS}

ANDRADE FF; MELO PCT; MORO JR. 2003. Seleção massal em duas populações de cenoura do tipo 'Brasília'. In: CONGRESSO BRASILEIRO DE OLERICULTURA, 43. Resumos... Recife: ABH (CD-ROM).

CAVALCANTI JA. 1998. Recomendações de adubação para o estado de Pernambuco $\left(2^{\circ}\right.$ aproximação). Recife: IPA. 198p.

CLEMENTE FMVT; RESENDE FV; VIEIRA, JV. 2006. Desempenho de cultivares e populações de cenoura em cultivo orgânico no Distrito Federal. In: CONGRESSO BRASILEIRO DE OLERICULTURA, 46. Resumos... Goiânia: ABH (CD-ROM).

CRUZ CD; REGAZZI AJ; CARNEIRO PCS. 2004. Modelos biométricos aplicados ao melhoramento genético. Viçosa: UFV. 480p.

EMBRAPA - Empresa Brasileira de Pesquisa Agropecuária. 2008, 10 de janeiro. Hortaliças em números. Disponível em http://www.cnph. embrapa.br/

LANA MM; VIEIRA JV. 2000. Fisiologia e manuseio pós-colheita de cenoura. Brasília: Embrapa Hortaliças. 15p. (Circular Técnica, 21).

LOPES WAR; NEGREIROS MZ; TEÓFILO TMS; ALVES SSV; MARTINS CM; NUNES GHS; GRANGEIRO LC. 2008. Produtividade de cultivares de cenoura em diferentes densidades de plantio. Revista Ceres 55: 482-487.

MCGUIRE RG. Reporting of objective color measurements. 1992. HortScience 27: 12541255.

OLIVEIRA AP; ESPÍNOLA FEJ; ARAÚJO JS; COSTA CC. 2001. Produção de raízes de cenoura cultivadas com húmus de minhoca e adubo mineral. Horticultura Brasileira 19: 77-80.

OLIVEIRA CD; BRAZ LT; BANZATTO DA. 2008. Adaptabilidade e estabilidade fenotípica de cultivares de cenoura. Horticultura Brasileira 26: 88-92.

OLIVEIRA EQ; BEZERRA NETO F; NEGREIROS MZ; BARROS JÚNIOR AP. 2004. Desempenho agroeconômico do bicultivo de alface em sistema solteiro e consorciado com cenoura. Horticultura Brasileira 22: 712-717.

PEREIRA JMSTK; MINIM VPR; PUSCHMANN R; VANETTI MCD; SOARES NF F; MORETTI CL; VIEIRA JV. 2008. Qualidade físico-química de mini-cenouras revestidas. Revista Ceres 55: 537-542.

PIMENTEL MS; LANA AMQ; DE-POLLI H. 2009. Rendimentos agronômicos em consórcio de alface e cenoura adubadas com doses 
crescentes de composto orgânico. Revista Ciência Agronômica 40: 106-112.

SCOTT AJ; KNOTT MA. 1974. A cluster analysis method for grouping means in the analysis of variance. Biometrics 30: 507-512.

SILVA RG; MIRANDA GV; CRUZ CD; GALVÃO JCC; SILVA DG. 2008. Potencial genético das populações de milho UFVM 100 e UFVM 200 avaliadas em solos com deficiência de nitrogênio. Revista Caatinga
21: 22-29.

STEEL RGD; TORRIE JH. 1981. Principles and procedures of statistics: a biometrical approach. New York: Mc-Graw Hill. 633 p.

VIEIRA JV; CRUZ CD; NASCIMENTO WM; MIRANDA JEC. 2005. Seleção de progênies de meio-irmãos de cenoura baseada em características de sementes. Horticultura Brasileira 23: 44-47.
VIEIRA JV; SILVA GO. 2008. Número mínimo de famílias para avaliação de parâmetros de cor em cenoura. Brasília: Embrapa Hortaliças. 24p. (Boletim de Pesquisa e Desenvolvimento, 41).

VILELA NJ; BORGES IO. 2008. Retrospectiva e situação atual da cenoura no Brasil. Brasília: Embrapa Hortaliças. 10p. (Circular Técnica, 59). 